\title{
2-D parametric analysis of Gas-Liquid Contacting in a Spinning Spiral Contactor
}

\author{
Ahmed A. Ayash \\ Materials Engineering Department, Faculty of Engineering, Mustansiriyah University \\ Baghdad, Iraq \\ ahmedayash@uomustansiriyah.edu.iq
}

\begin{abstract}
The paper is about a parametric study of counter-current gas-liquid flow in a spinning spiral channel. The study is conducted by solving numerically the governing equations for mass, momentum and species using an interface shape predicted independently. The case of a dilute solute is considered in all the compautaions. The purpose of this study is to examine, for the first time, the role of three key parameters: rotation rate, channel aspect ratio and flow rate of both phases. Interestingly, the results demonstrated that by adjusting the rotation rate $(\Omega)$, the contacting process could be optimised. For a range between 1000 and $20,000 \mathrm{rpm}$, it was found that $\Omega$ $=16,000 \mathrm{rpm}$ gave a maximum mass transfer coefficient. Furthermore, the data showed that the spiral performance was enhanced considerably by changing the channel aspect ratio. Reducing the channel width from $4 \mathrm{~mm}$ to $1 \mathrm{~mm}$ increased the mass transfer coefficient by a factor of two. Finally, at a given rotation rate and channel aspect ratio, an improvement in mass transfer was observed by adjusting the flow rates of the contacting phases. Increasing the flow rate of both phases increased the mass transfer coefficient also by a factor of two.
\end{abstract}

Keywords: Spinning spiral contactor, Parametric analysis, Mass transfer, Coriolis motion, Rotation rate, Channel aspect ratio.

\section{Introduction}

Gas-liquid contacting is widely encountered in various industrial and analytical applications such as removing species from a gas stream (gas-absorption), purifying a liquid stream (desorption) and separation of fluid-phases (distillation). The rotating spiral technique is an emerging approach that can handle any two immiscible fluids [1]. This distinctive feature allows a variety of mass transfer applications to be within the capability of this technique, including gas-liquid contacting. In this technique, the mechanism of contact of fluid phases avoids any mixing, providing solutions for most of the difficulties arising as a result of dispersion of one phase into the other. The technique, simply, uses a spiral channel spinning around its axis, producing both centrifugal and Coriolis acceleration. The centrifugal effect enables the fluid phases to flow side by side as two separate layers (Fig. 1.1), either counter-currently or co-currently depending on the magnitude of the pressure gradient along the channel and the rotation rate. The Coriolis acceleration and the variation of streamwise velocity in the cross-sectional flow area produce Corilois secondary motion in each phase (as those shown in Fig. 1.1) that can enhance mass transfer by convection. In contrast with other dispersed-phase techniques, this organised pattern of contacting can be achieved with a high degree of control. The relative flow rates and the hydrodynamics obtained (i.e. phase layer thicknesses and phase velocities) are decoupled and can be controlled independently with this technique. The former, which is determined mainly by phase equilibrium characteristics, governs the extent of separation. The latter, on the other hand, dictates the separation rate. Therefore, with the ability to control these parameters independently, optimum contacting of systems having different equilibrium and transferring properties can be achieved, in principle, using the rotating spiral technique $[2,3]$.

The first theoretical investigation of rotating spiral contacting appears to be a study of Mochalova et al. [4]. The authors looked at the hydrodynamic characteristics and the mass transfer performance in a rotating spiral channel, considering a stable laminar flow. This modelling work was extended by Zhavoronkov et al. [5] to include the behaviour of flow and the mass transfer rate at the entrance region of the spiral. In either case, the solution of the governing equations of motion was approximated for the case of external flow over a rotating spiral surface to find the liquid layer thickness and mass transfer coefficient in the liquid side. Unfortunately, there is no indication in the literature that these theoretical works were ever utilized or even compared with experimental work. Thus, the accuracy of these models was not proved. Further, approximating the solution to the case of external flow means the effect of end-walls, surface tension, and interfacial shear stresses were not considered. All these factors affect directly the shape and size of phase layers [2]. Thus, a reliable prediction 
of the liquid layer thickness and mass transfer coefficient using these models is not expected in general. Ortiz-Osorio et al. [6] performed successfully a numerical computation for a specific experimental condition of rotating spiral distillation. In the work, the interface is assumed to be a perfect circle which may not be a feasible assumption for large scale spiral channels. Subsequently, a theoretical study has been reported in MacInnes et al. [7]. The study included analysis and modelling of twophase contacting in a rotating spiral with infinite-width. As a result, an analytical model 'wide-channel model' was developed. Although the wide channel model is helpful in exploring some aspects of the rotating spiral contacting, it does not lead to a full understanding of two-phase contacting in actual channels. The assumption of infinite width (i.e. no endwalls) makes the model unable to capture the effect of the interface shape and Coriolis secondary motion. To understand the mechanism of mass transfer in rotating spiral contacting, it is important to consider the actual interface shape and the role of the secondary motion since they affect directly the mass transfer. Such level of modelling has been presented recently in Ayash and MacInnes [8]. In the work, a 2-D numerical solution of the governing equations using an interface shape calculated independently was developed and demonstrated.

Here, the effectiveness of the 2-D model is examined, critically, by comparing its predictions to a wide of range experiments data available in the relevant literature, determining its limitations. Further, a parametric study is carried out where desorption of acetone from water into air was taken a reference case. The purpose of the study is to explore the effect of three key parameters: the rotation rate, channel aspect ratio and phase flow rates. The effect of these parameters has not been studied experimentally and it is important to understand their role in gas-liquid contacting. It is believed that such study will help to understand the rotating spiral technology and to demonstrate its flexibility.



Fig. 1: Mechanism of secondary motion formation in a two-phase contacting counter-currently in a rotating spiral channel. $u, v$ and $w$ are the velocity components in $x, y$ and $z$ directions, respectively. (a) Coriolis forces and (b) illustrative structure of secondary motion.

\section{Governing Equations}

In the spiral, the motion and the mass transfer of solute in each phase are governed by exactly the same equations but with the physical property values (i.e. density, $\rho$, viscosity, $\mu$, diffusivity, $D$ and molar density, $n$ ) those for the particular phase. These equations can be expressed in terms of the velocity components shown in Fig. 1, the solute mole fraction $(Y)$ and the piezometeric pressure $(p)$ as follows:

Continuity Equation:

$$
\frac{\partial u}{\partial x}+\frac{\partial v}{\partial y}=0
$$

Momentum Equations:

$$
\begin{gathered}
\rho u \frac{\partial u}{\partial x}+\rho v \frac{\partial u}{\partial y}=-\frac{\partial p}{\partial x}+\mu\left(\frac{\partial^{2} u}{\partial x^{2}}+\frac{\partial^{2} u}{\partial y^{2}}\right) \\
\rho u \frac{\partial v}{\partial x}+\rho v \frac{\partial v}{\partial y}=-\frac{\partial p}{\partial y}+\mu\left(\frac{\partial^{2} v}{\partial x^{2}}+\frac{\partial^{2} v}{\partial y^{2}}\right)+\underbrace{2 \rho \Omega w}_{\text {Coriolis term }}
\end{gathered}
$$


Species Equation:

$$
\begin{gathered}
\rho u \frac{\partial w}{\partial x}+\rho v \frac{\partial w}{\partial y}=-\frac{d P_{B}}{d z}+\mu\left(\frac{\partial^{2} w}{\partial x^{2}}+\frac{\partial^{2} w}{\partial y^{2}}\right)+\underbrace{\rho R \Omega^{2} \sin \alpha}_{\text {Centrifugal lenriolis term }}-\underbrace{2 \rho \Omega v} \\
n u \frac{\partial Y}{\partial x}+n v \frac{\partial Y}{\partial y}=-n w \frac{d Y_{B}}{d z}+n D\left(\frac{\partial^{2} Y}{\partial x^{2}}+\frac{\partial^{2} Y}{\partial y^{2}}\right)
\end{gathered}
$$

It is important to note here that the above equations describe steady, incompressible and fully developed flow of two phases contacting in a 2-D section along the channel where heat transfer has no effect and a dilute solute being transfer between the two phases. Under these conditions, the pressure drop along the channel is taken to be constant $\left(d P_{B} / d z\right)$ and the change in solute mole fraction in that same direction is replaced by the change in the bulk mole fraction $\left(d Y_{B} / d z\right)$. Further, since the channel, typically, has a small curvature ratio, it is taken to be straight and inclined by $\alpha$ angle relative to its tangential direction. More information regarding the equations formulation, the justifications of these approximations, the interface shape calculations and the numerical solution can be found in details in Ayash and MacInnes [8] and Ayash [9].

At the interface, the governing equations in each phase are coupled by the continuity of velocity, mass transfer flux and solute concentration. Also, for gas-liquid contacting, the interface has a static shape at a given rotation rate and does not depend on the phase flow rates or the interface position [1]. This imposes the sum of velocity components in the $x$ and $y$ directions to be zero (kinematic condition). These boundary conditions can be expressed for either phase in vector forms by selecting a unit vector normal to the interface surface and pointing to the liquid phase $(\vec{n})$. So, using ' $L$ ' to designate the heavy phase region and ' $V$ ' the light phase region, the boundary conditions can be written as follows.

$$
\begin{gathered}
\vec{n} \cdot \vec{V}_{V}=\vec{n} \cdot \vec{V}_{L}, \quad n_{V} D_{V}\left(\vec{n} \cdot \nabla Y_{V}\right)=n_{L} D_{L}\left(\vec{n} \cdot \nabla Y_{L}\right) \quad \text { and } \quad Y_{V}=f^{\prime} Y_{L} \text { (Equilibrium relation) } \\
n_{x} u_{V}+n_{y} v_{V}=n_{x} u_{L}+n_{y} v_{L}=0 \text { (kinematic condition) }
\end{gathered}
$$

where $f^{\prime}$ is the solute equilibrium distribution.

The typical conditions at the channel walls, which are non-adsorbing and fixed, are no-slip velocity and zero mass transfer flux of solute. These further conditions are then:

$$
\vec{n} \cdot \vec{V}=0 \quad \text { and } \quad \vec{n} \cdot \nabla Y=0
$$

Additionally, the overall balance on the solute constraints the bulk concentration gradient in the gas side in terms of that for the liquid side:

$$
-n_{V} Q_{V} \frac{d Y_{V B}}{d z}=n_{L} Q_{L} \frac{d Y_{L B}}{d z}
$$

where $Q_{V}$ and $Q_{L}$ are the gas and the liquid volume flow rates, respectively.

\section{Model Predictions}

The first extensive mass transfer data of a gas-liquid contacting in a spiral channel have been reported in MacInnes and Ayash [3] and Ayash [9]. The authors investigated desorption of four different organic solutes from water into air at 24, 30 and $49^{\circ} \mathrm{C}$ in a spiral channel with $1.5 \mathrm{~mm}$ height (parallel to y-axis), $4 \mathrm{~mm}$ width (parallel to $\mathrm{x}$-axis) and $R \sin \alpha=\quad 5.57$ $\times 10^{-4} \mathrm{~m}$, and rotates at $3200 \mathrm{rpm}$. This range of temperatures gives seven phase and solute systems in terms of $f^{\prime}$ (equilibrium constant) and changes the phase and solute physical properties. The experimental measurements of those works are compared now with the 2-D model predictions. To simplify the comparison, the interface shape is calculated using the intermediate radial position $(25 \mathrm{~mm})$ [8]. Further, the value of the surface tension is taken to be that of pure water at $25^{\circ} \mathrm{C}$ $(0.07 \mathrm{~N} / \mathrm{m})$. The other physical properties used in the interface calculations and the 2-D model predictions are phase densities, viscosities, molar densities and diffusion coefficients and those are given in details in [3,9]. Finally, the contact 
angles are needed and they are taken as those for the air and water contacting in the same experimental channel [1]. These values are consistent with those reported in the relevant literature[10,11].

Figs. 2 and 3 below show the experimental mass transfer coefficients (grey symbols) measured at a gas phase flow rate of $3 \mathrm{NL} / \mathrm{min}$ along with the corresponding 2-D predictions (red symbols). The data are plotted against the purification factor which is the product of the equilibrium constant $\left(f^{\prime}\right)$ and phase flow rate ratio $\left(q_{n}=Q_{V} / Q_{L}\right)$. The result of wide-channel model (WCM) developed by MacInnes et al. [7] is also included and it is shown as a dashed curve.

In general, for the different phase and solute systems, the results in Figs. 1 and 2 show that the 2-D computation captures convincingly the mass transfer performance over a large range of the experimental data while WCM failed to predict, quantitatively, the experiments. This is, perhaps, expected since WCM does not take into account the interface shape and the presence of Coriolis secondary motion while the 2-D model does. Also, the results show that there is a deviation between the 2-D model predictions and the experiments. In particular, the 2-D model over-predicted $K_{L} a$ values at a low liquid phase flow rate (or large $-f^{\prime} q_{n}$ ) for the systems with large $f^{\prime}$ (3.38 and 5.5). It is likely that the minor deviations observed are mainly the result of interface shape approximations and experimental uncertainties. This assessment is examined here by repeating the computations for $f^{\prime}=3.83$ and 5.5 with an interface shape has a relatively larger meniscus height. The results with larger meniscus height are shown in Figs. 2 and 3 as circles with fainter shade. As can be seen, the small change in the meniscus height brings the model closer to the experiment at large $-f^{\prime} q_{n}$. This model sensitivity analysis using an interface shape with a larger meniscus size suggested that the difference is likely due to the approximations used in the interface shape calculations, as expected. One of these approximations is using contact angles measured at static conditions. In practice, these angles might be more sensitive to other processes, such as evaporation, during the contacting process. Any changes in the contact angles affect the meniscus size, which is a crucial parameter in determining the mass transfer at low liquid phase flow rates. However, without adjusting the contact angles and using a local radial position $(25 \mathrm{~mm})$ and water surface tension $(0.07 \mathrm{~N} / \mathrm{m})$, the 2-D model predicts closely the data, especially over $-f^{\prime} q_{n}$ between 1 and 4 which are typical for desorption process.

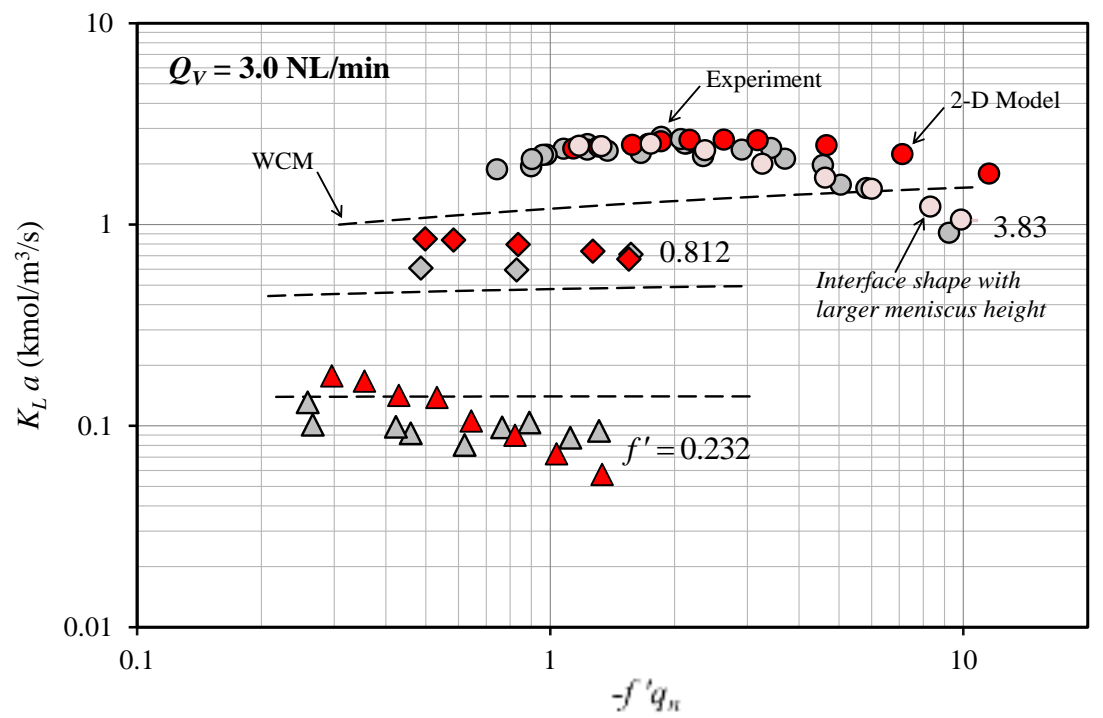

Fig. 2: Overall volumetric mass transfer coefficients for three $f^{\prime}$ values $\left(0.232,0.812\right.$ and 3.83) at $Q_{V}=3.0 \mathrm{NL} / \mathrm{min}$. The grey symbols are the experiments and the red ones are the 2-D model predictions at the corresponding conditions. The dashed lines are the wide channel model results. 


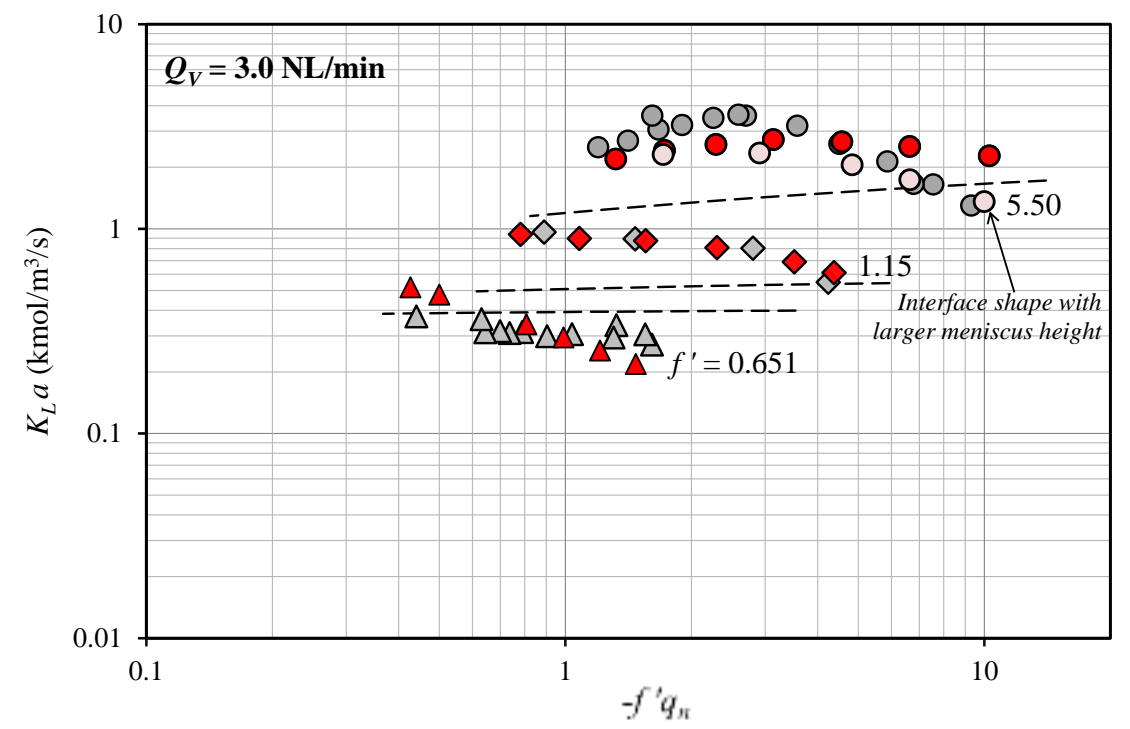

Fig. 3: As in Fig. 2 but for $f^{\prime}=0.651,1.15$ and 5.5. Again, the dashed lines are the wide channel model results at the corresponding conditions and the shaded symbols are the experimental points (grey points) and the 2-D results (red points).

\section{Model Parametric Analysis}

The comparisons made in the previous sections show clearly the capability of the 2-D computational model to predict over a wide range of experimental conditions. So it can be employed now to demonstrate the effect of some key parameters which have not been tested experimentally in the previous works. Three main parameters are considered in this study: rotation rate, channel aspect ratio and different phase flow rates. To simplify the task, desorption of acetone from water into air is taken as a reference case $\left(f^{\prime}=1.15\right)$. The properties of this system used in the computations are given in [3] and [9]. Further, the purification factor $\left(-f^{\prime} q_{n}\right)$ is fixed at 1.2 which is not expected to be unreasonable value for desorption process.

\subsection{Different Rotation Rate}

One of the most key parameters that this technology is based on and is fully under external control is the rotation rate. The effect of rotation rate on the mass transfer process is examined here by computing for a range from 1000 to $20,000 \mathrm{rpm}$ with the phase flow rates held constant at $Q_{V}=3 \mathrm{NL} / \mathrm{min}$ and $Q_{L}=2.2 \mathrm{~mL} / \mathrm{min} \quad\left(-f^{\prime} q_{n}=1.2\right)$. This has been done by adjusting both the liquid layer thickness and $d P_{B} / d z$ until the computed flow rates are correct. Fig. 4 shows the computed streamwise velocity, secondary flow and solute mole fraction distribution for two extreme values. In each contour plot, the colours are shaded from blue through green to red corresponding to the range of values from minimum to maximum. Also, it is important to note that the vector plots, which reveal the secondary flow, are plotted with an arrow length is proportional to the transverse velocity magnitude and using the same scale factor in the two cases below for each phase. 



$=1000 \mathrm{rpm}$
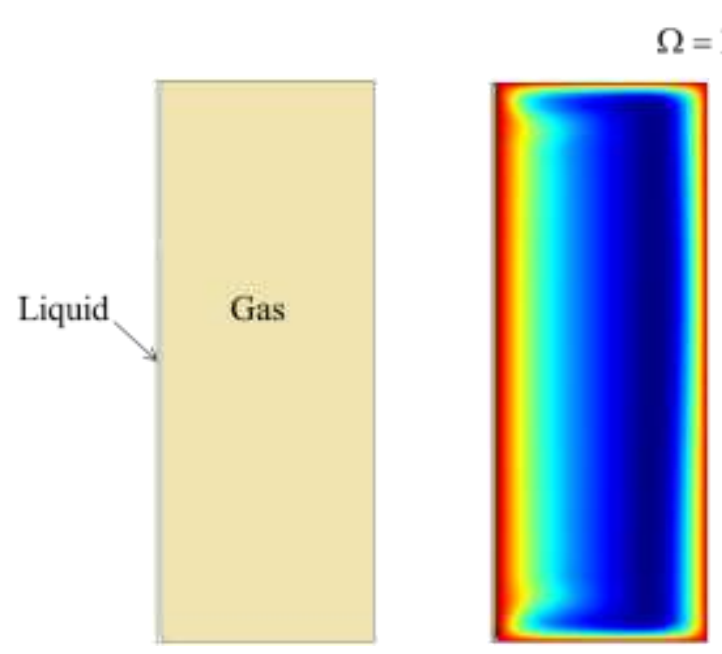

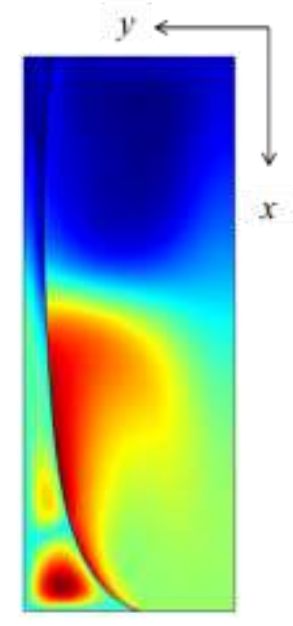

Fig. 4: Numerical results (streamwise velocity, secondary flow and the solute mole fraction distribution) for rotation rates of 1000 and $16000 \mathrm{rpm}$ at $Q_{V}=3 \mathrm{NL} / \mathrm{min}$ and $Q_{L}=2.2 \mathrm{~mL} / \mathrm{min}\left(-f^{\prime} q_{n}=1.2\right)$.

First of all, the effect of increasing the rotation rate on both the interface shape and thickness of the phase layers is evident in Fig. 4. As increasing $\Omega$ both the transverse centrifugal component $\left(R \Omega^{2} \cos \alpha\right)$ which determines the interface shape and the longitudinal component $\left(R \Omega^{2} \sin \alpha\right)$ that drives the liquid phase increase. So in the figure, the meniscus height, the interface tilt and the liquid layer thickness decrease simultaneously with the rotation rate, as expected.

Further, the effect of the rotation rate on the pattern of streamwise velocity, the strength of the secondary motion and hence the solute mole fraction distribution is also clear in Fig. 4. Considering the gas phase first, increasing $\Omega$ increases the level of the Coriolis terms in $v$ and $w$ equations. Accordingly, the strength of Coriolis secondary motion and the distortion of the gas streamwise velocity profile become more pronounced with increasing the rotation rate. In the liquid layer, on the contrary, the streamwise velocity increases while the secondary motion diminishes as rotation rate increases. This, perhaps, is not surprising since increasing the rotation rate is associated with a sharp decrease in the liquid layer thickness. As the layer thickness decreases, the viscous effect becomes important relative to the Coriolis effect and hence a weaker Coriolis motion generates.

Looking now at the mass transfer coefficient, the variation in mass transfer performance with the rotation rate can be quantified. Fig. 5 below gives the values of the overall volumetric mass transfer coefficients over the tested range of rotation rate. It is important to note that the maximum change in the interfacial area per unit volume $(a)$ associated with the change in the interface shape is about 5\%. So the behaviour shown in Fig. 5 is essentially due to the change in $K_{L}$ values. The data show clearly that the mass transfer performance increases rapidly with the rotation rate up to about $\Omega=8000 \mathrm{rpm}$. This is 
attributed to the sharp decrease in the liquid layer thickness and the noticeable increase in the strength of the gas phase secondary flow (Fig. 4) with the rotation rate which together lead to enhancing the mass transfer in the channel. Beyond $\Omega$ $=8000 \mathrm{rpm}$, however, the mass transfer responds weakly to the change in the rotation rate: increasing slightly and then decreasing and thus forming a broad peak with a maximum at around $\Omega=16,000 \mathrm{rpm}$.



Fig. 5: Computed overall mass transfer coefficients against the rotation rate. The flow conditions as in Fig. 4.

\subsection{Different Channel Aspect ratio}

The channel aspect ratio may affect the mass transfer performance and is examined here by varying $W$ (the channel dimension parallel to the phase layers). Varying only $W$ and keeping $h$ constant ensures approximately a constant interfacial area per unit volume $\left(a \approx h^{-1}\right)$ for the tested cases. Therefore, any changes in mass transfer would be a direct result of changes in $K_{L}$ values. A channel with three different widths are simulated: $W=1,2$ and $3 \mathrm{~mm}$ in addition to that used in the original design ( $4 \mathrm{~mm}$ ). The standard set of plots for each case is shown in Fig. 6 where $\Omega=3200 \mathrm{rpm}$ and, again, the phase flow rates are held constant at $Q_{V}=3 \mathrm{NL} / \mathrm{min}$ and $Q_{L}=2.2 \mathrm{~mL} / \mathrm{min}\left(-f^{\prime} q_{n}=1.2\right)$. 
$W=4 \mathbf{m m}$

$W=3 \mathbf{~ m m}$
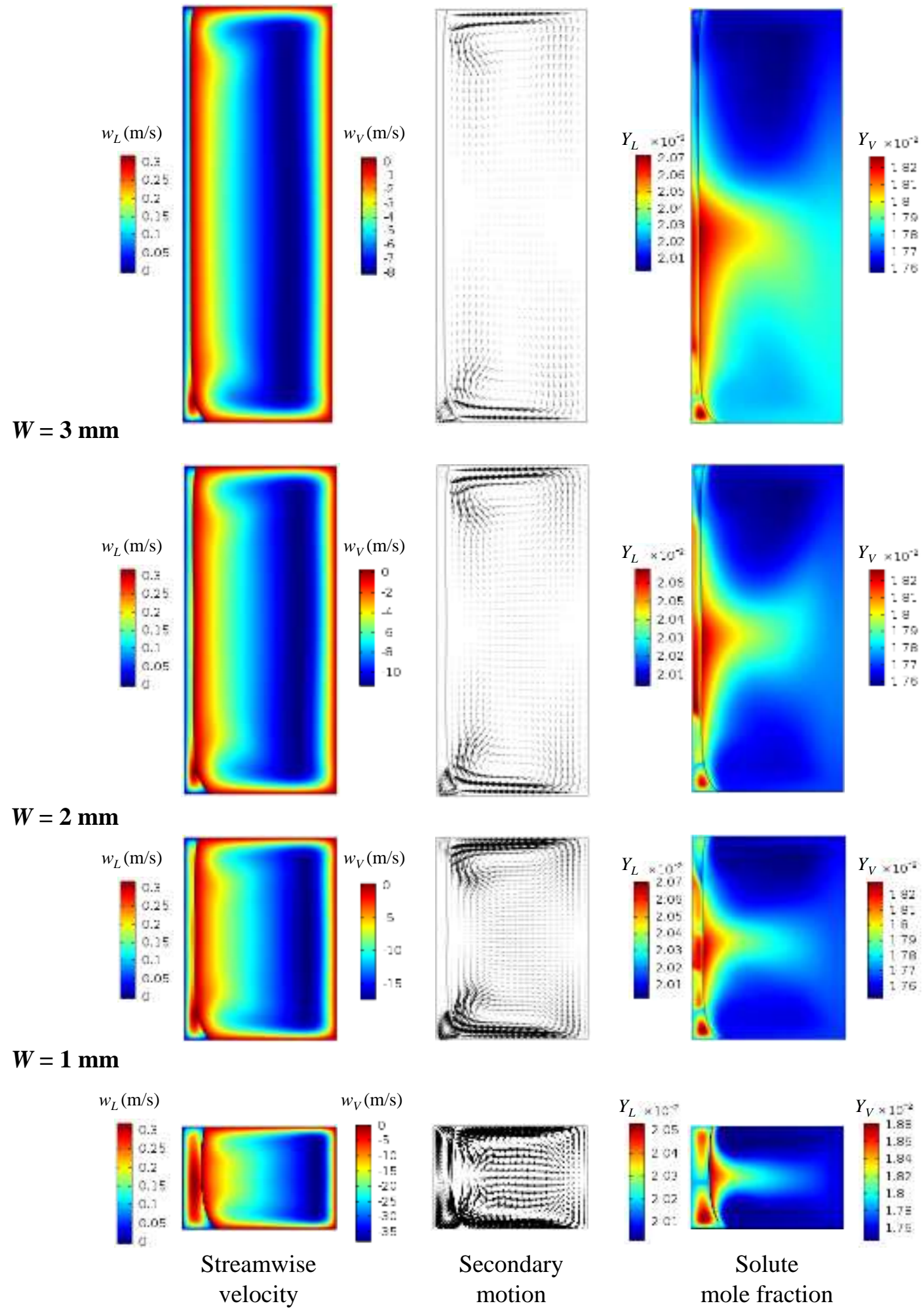



Secondary motion

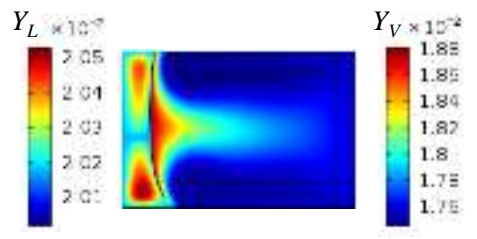

Solute mole fraction

Fig. 6: Effect of different channel aspect ratio. The conditions are $Q_{V}=3 \mathrm{NL} / \mathrm{min}, Q_{L}=2.2 \mathrm{~mL} / \mathrm{m}\left(-f^{\prime} q_{n}=1.2\right)$ and $\Omega=3200$ rpm. 
It is clear in Fig. 6 that the variation in channel width has a strong influence on the main features of the contacting process. First of all, reducing the channel width increases the interfacial surface force to the centrifugal force. This results in a more curved interface between the phases. Further, the variation in channel width affects directly the character of the main flow and the Coriolis secondary motion and hence the solute distribution in both phases. For the liquid phase, decreasing the channel width results in a thicker liquid layer to conserve the mass and hence allows to the same amount of liquid to flow in a smaller channel. So, for example, the fraction of liquid phase increases about 2.5 times as $W$ decreases from 4 to $1 \mathrm{~mm}$. As a direct consequence of that, the difference between the centre of the layer and its ends becomes minor as $W$ decreases. Thus, at $W=1 \mathrm{~mm}$, the liquid phase flows approximately evenly everywhere, causing a strong variation in streamwise liquid velocity. The effect of streamwise liquid velocity distribution on the Coriolis motion is evident in Fig. 6. Since the variation in streamwise velocity under rotation causes the generation of the secondary flow, it is natural to see that the rotation of the smaller channel gives rise to stronger vortices occupying most the liquid passage relative to those with sufficiently large channel width.

It is also clear in Fig. 6 that the gas phase Coriolis motion becomes more active in the smaller channel. This is mainly due to the large increase in the gas phase velocity associated with the large reduction in the size of the passage available for the same phase.

Quantitative information about the solute mass transfer is shown in Fig. 7. The figure shows the molar flux of the solute along the interface for the four different channels. The results in the figure indicate clearly that the solute flux increases as the channel width decreases. The ratio of the flux at corner relative to that at the middle of the layer increases markedly as $W$ decreases. This is certainly because of the pronounced evolution in the secondary motion noticed in both phases in Fig. 6 , which appears to have a strong influence on mass transfer in the small channel. This effect is confirmed by looking at the mass transfer coefficient values. Fig. 8 shows these values against the channel width. As expected, the transfer coefficient is proportional inversely with the channel size, reaching the maximum at $W=1 \mathrm{~mm}$.

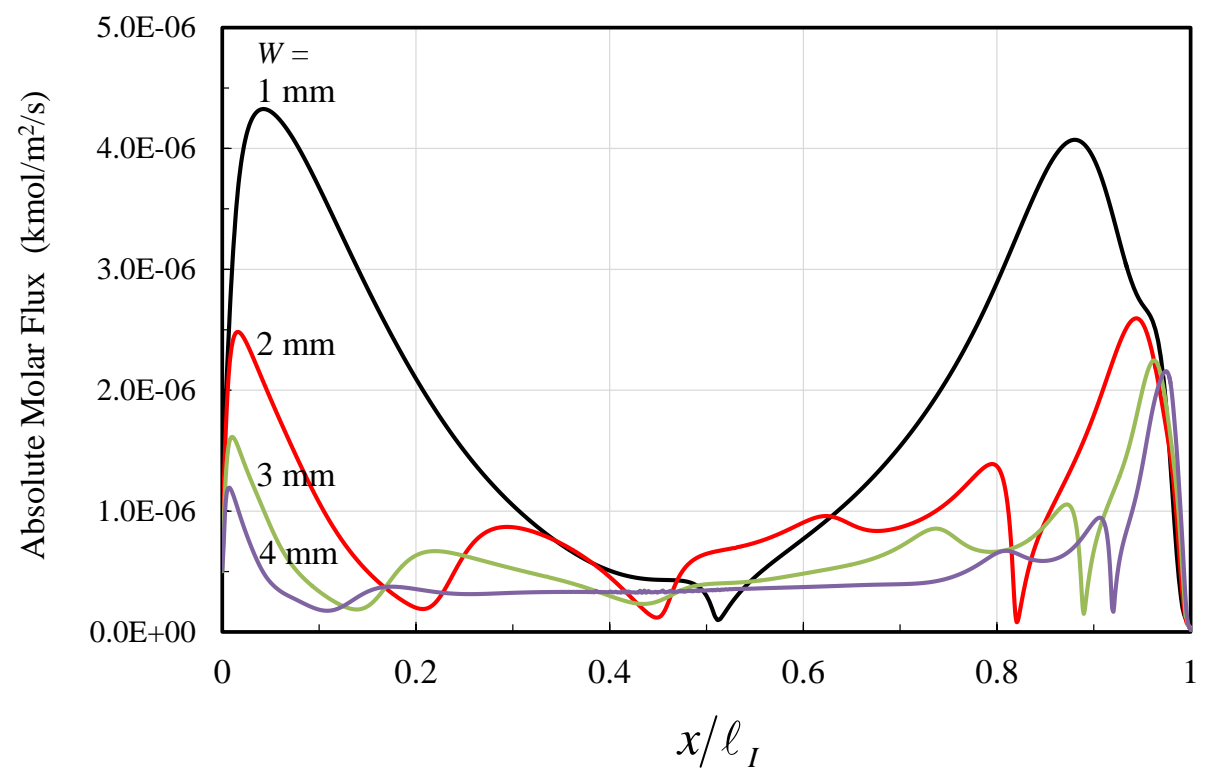

Fig. 7: Solute molar flux along the phase interface for channel with different widths where $\ell_{I}$ is the interface length and the conditions as in Fig. 6. 


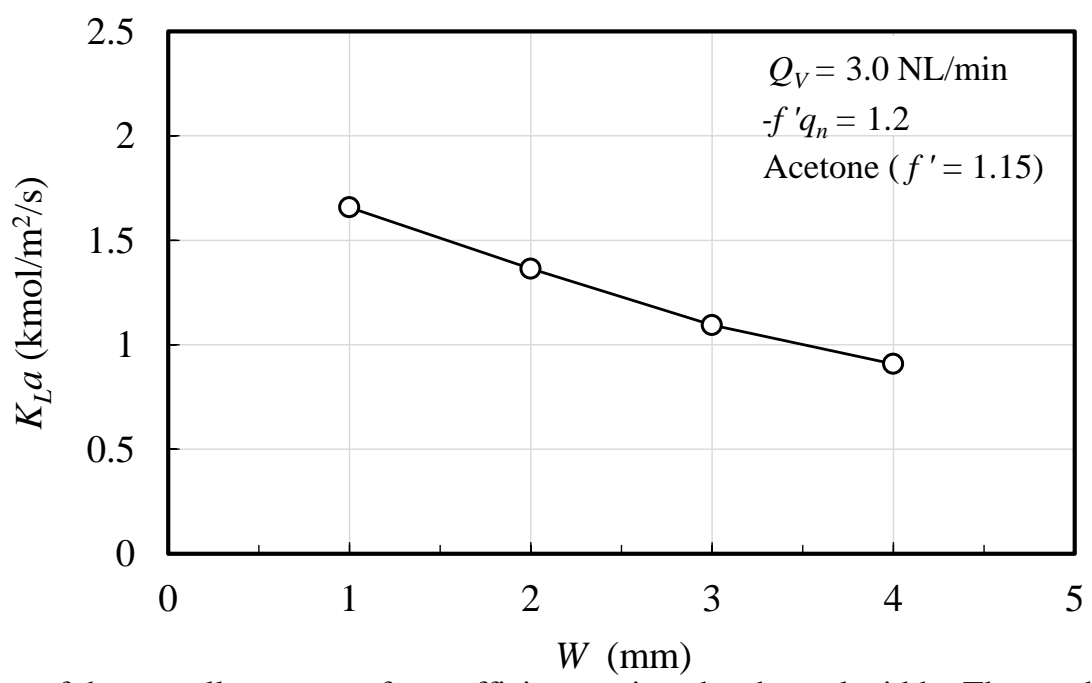

Fig. 8: Values of the overall mass transfer coefficient against the channel width. The conditions as in Fig. 6.

\subsection{Different Phase Flow Rate}

The experiments carried out in MacInnes and Ayash [3] and Ayash [9] are based on fixing the flow rate of one of the phases, usually the gas phase, and change the other. However, it is important to examine how varying these parameters together affects the process of contacting. Fig. 9 below shows the sensitivity of the individual transfer coefficients along with the overall coefficient to different values of gas and liquid flow rates. Again, the purification factor $\left(-f^{\prime} q_{n}\right)$ and $f^{\prime}$ are fixed at 1.2 and 1.15, respectively, for all the cases. Also, before discussing the results, it is worth mentioning that the range of gas and liquid flow rates shown in Fig. 9 corresponds to a range of Reynolds number of $\operatorname{Re}_{L} \approx 3-24$ and $\operatorname{Re}_{V} \approx 270-2150$.

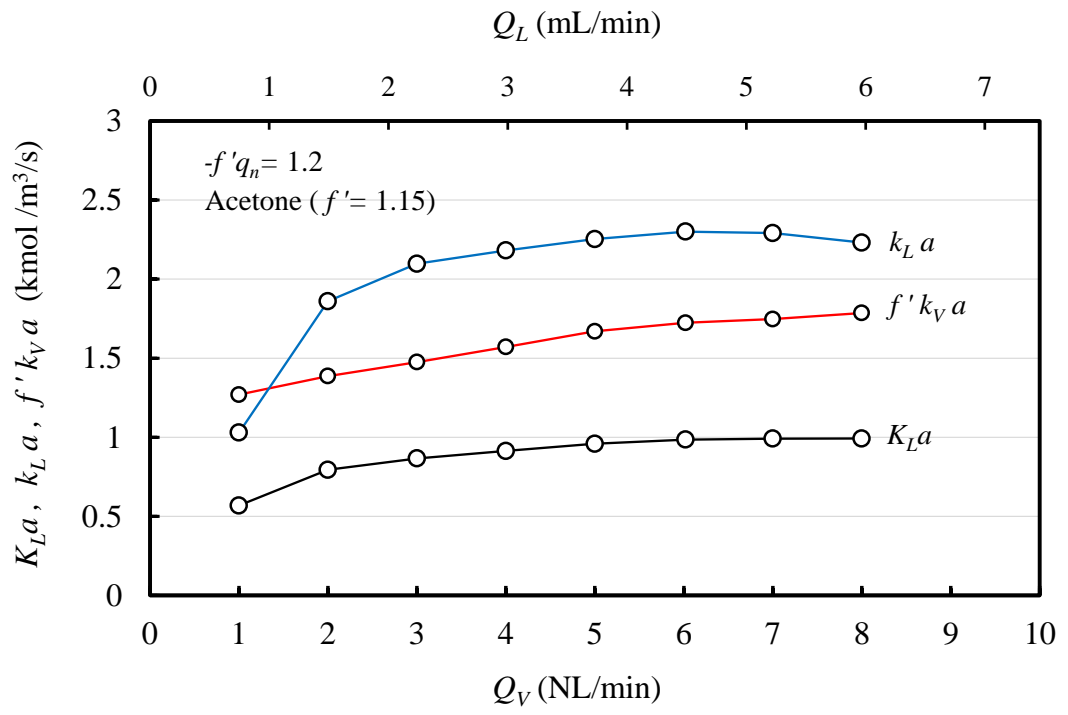

Fig. 9: Mass transfer coefficients over different values of gas and liquid flow rates. The other conditions are $f^{\prime}=1.15,-f^{\prime} q_{n}=1.2$ and $\Omega=3200 \mathrm{rpm}$.

Considering the results of the gas phase first (red curve), the behaviour is immediately evident: $f^{\prime} k_{V} a$ increases gradually as the gas phase flow rate increases. There is no doubt that this effect is mainly due to increasing Reynolds number (ratio of convective effect to the viscous effect) which, in turn, results in a stronger Coriolis motion, enhancing mass transfer by convection. On the other hand, the solute mass transfer in the liquid side (blue curve) reveals an interesting behaviour. 
It can be seen clearly that $k_{L} a$ increases as the liquid flow rate increases (labels on the upper abscissa) and then starts decreasing at around $Q_{L}>4.5 \mathrm{~mL} / \mathrm{min}$, forming a wide peak. This behaviour can be interpreted in terms of both the liquid layer thickness and the combined effect of the main flow and secondary flow. It must be clear now that at a low liquid flow rate, the interface shape imposes a situation where the diffusion length scale is large at the ends compared to that at the middle of the layer (due to the menisci formation) and a large fraction of the fluid is diverted there. Also, it must be known that small liquid flow rate means a weak secondary flow generated in the menisci regions. So the rapid increase in $k_{L} a$ with the liquid flow rate (from 0.7 to about $3 \mathrm{~mL} / \mathrm{min}$ ) is mainly because the menisci effect is minimised along with the expected increase in secondary motion with the liquid flow rate. The variation in menisci effect with the liquid phase flow rate can be seen clearly in Fig. 10 through the progression in solute mole fraction distribution. But increasing the liquid flow rate has an opposite effect as well. It leads to increasing both the layer thickness and hence the diffusion length scale and the axial mass transfer by the bulk flow. Thus, any dropping in mass transfer due to increasing the diffusion distance and the bulk flow appears to be over-compensated by the increased effect of secondary motions and the solute difference across the flowing fluid. This likely explains the continuous increase in $k_{L} a$ observed in Fig. 10, reaching an optimum at $Q_{L}$ around $4.5 \mathrm{~mL} / \mathrm{min}$. Further increasing the liquid phase flow rate, however, the mass transfer will be more affected by the decreasing effect of liquid layer thickness and the axial mass transfer along the channel and hence $k_{L} a$ decreases.

The overall mass transfer coefficient is a direct result of the variation in the individual coefficients seen in Fig. 9, particularly $f^{\prime} k_{V} a$. Accordingly, $K_{L} a$ increases first as both $k_{L} a$ and $f^{\prime} k_{V} a$ increases up to $Q_{V}=5 \mathrm{NL} / \mathrm{min}$. Then, it remains approximately constant, following the same behaviour of $k_{L} a$ and $f^{\prime} k_{V} a$.
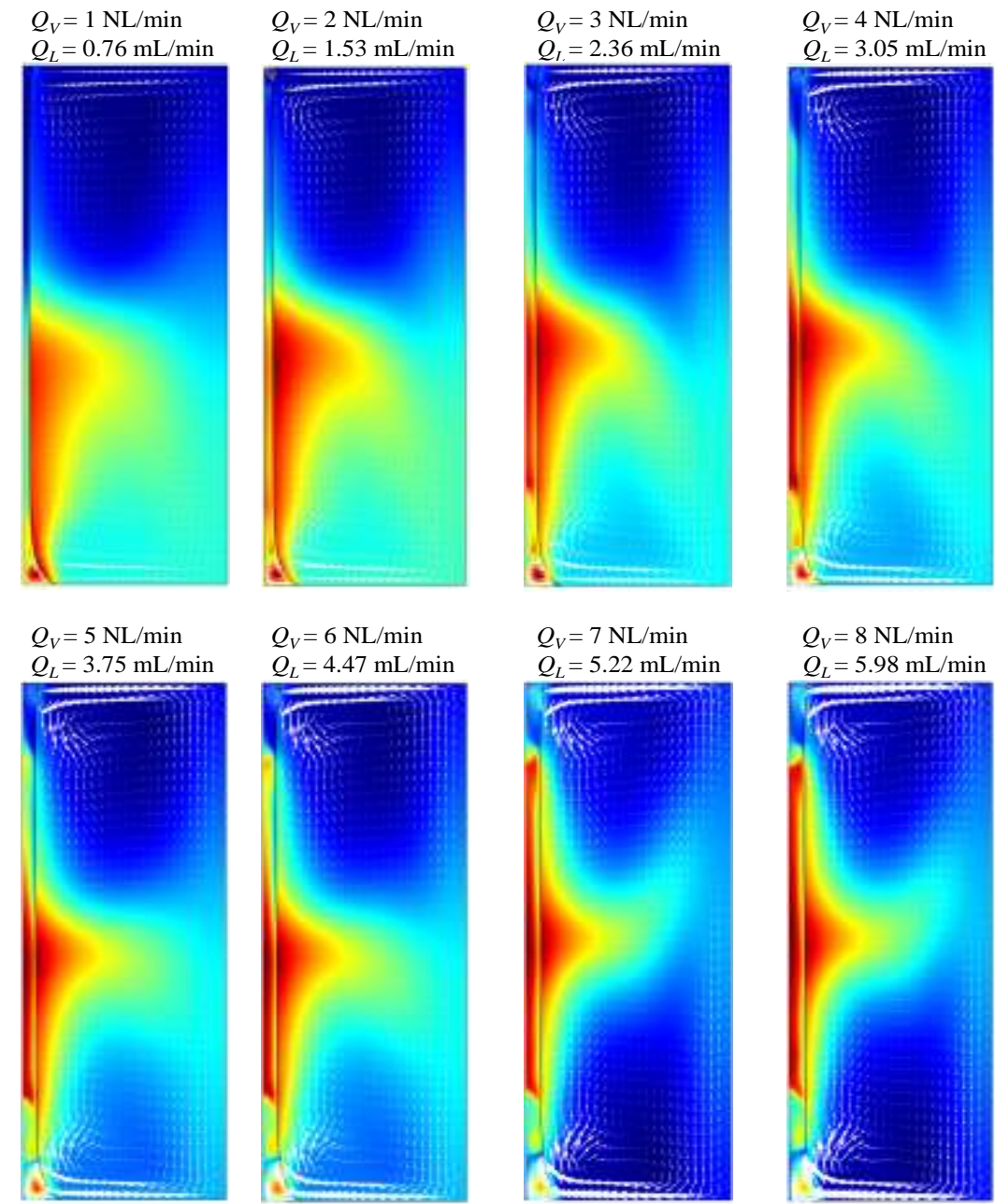

Fig. 10: Solute mole fraction distribution and Coriolis secondary motion at different liquid and gas phase flow rates. 


\section{Conclusion}

A parametric study using a 2-D model was presented. The main objective here is to investigate the effect of three key parameters: rotation rate, channel aspect ratio and both the gas and liquid phase flow rate. The effect of these parameters has not been studied experimentally and it is important to understand their role in gas-liquid contacting process. For this objective, desorption of acetone $\left(f^{\prime}=1.15\right)$ at $-f^{\prime} q_{n}=1.2$ and $\Omega=3200 \mathrm{rpm}$ was taken a reference case to carry out the study.

Interestingly, the 2-D numerical results showed that the channel geometry can be engineered and the rotation rate and phase flow rates can be adjusted to enhance the rotating spiral contacting. It was found that reducing the channel width from $4 \mathrm{~mm}$ to $1 \mathrm{~mm}$ increased the mass transfer coefficient approximately by a factor of two. This reduction in channel width changed the strength of Coriolis motion in both phases. It became stronger and occupied most the gas and liquid phase passage, resulting in improving the mass transfer. Further, the rotation rate, which is a key operating parameter, was examined over a range between 1000-20,000 rpm. The results showed that by adjusting the rotation rate, the contacting process could be optimised. By increasing the rotation rate, three main changes occurred: (1) the shape of the interface flattened, (2) the liquid layer thickness decreased and (3) the Coriolis motion in the gas phase increased. These three changes improved the mass transfer of acetone in both phases achieving a maximum mass transfer coefficient at around $\Omega=16,000$ $\mathrm{rpm}$. The other parameter examined was the flow rate of the contacting phases. The flow rate of the gas phase and the liquid

phase can be controlled simultaneously at a desired $-f^{\prime} q_{n}$ during operation. In practice, this can be done by simply adjusting a needle valve or pump setting. The 2-D model results showed that increasing the flow rate of both phases from $Q_{V}=1$ $\mathrm{NL} / \mathrm{min}$ and $Q_{L}=0.76 \mathrm{~mL} / \mathrm{min}$ to $Q_{V}=8 \mathrm{NL} / \mathrm{min}$ and $Q_{L}=5.98 \mathrm{~mL} / \mathrm{min}$ increases the mass transfer coefficient by about a factor of two. This was mainly attributed to the convective effect of Coriolis motion in the gas and liquid phase. Finally, this study provides evidence that the rotating spiral technique is a flexible approach and with simple adjustments to some operating and design parameters the separation processes can be optimised.

\section{References}

[1] J. M. MacInnes and M. K. S. Zambri, "Hydrodynamic characteristics of a rotating spiral fluid-phase contactor," Chem. Eng. Sci., vol. 126, pp. 427-439, Apr. 2015.

[2] J. M. MacInnes, J. Ortiz-Osorio, P. J. Jordan, G. H. Priestman, and R. W. K. Allen, "Experimental demonstration of rotating spiral microchannel distillation," Chem. Eng. J., vol. 159, no. 1-3, pp. 159-169, 2010.

[3] J. M. MacInnes and A. A. Ayash, "Mass transfer characteristics of rotating spiral gas-liquid contacting," Chem. Eng. Sci., vol. 175, pp. 320-334, 2018.

[4] N. S. Mochalova, L. P. Kholpanov, and V. Y. Shkadov, "Hydrodynamics and Mass Transfer in a Liquid Layer at a Rotating Surface., [Translated from Inzhenerno'-Fizicheskii Zhurnal, Vol, 25, No. 4, pp. 648-655; October, 1973] by Plenum Publishing Corpo," pp. 1251-1256, 1975.

[5] N. M. Zhavoronkov, V. A. Malyusov, N. S. Mochalova, and L. P. Kholpanov, "Mass transfer in a liquid layer of variable thickness on a rotating archimedes spiral taking account of the enterance region, [Translated from Zhurnal Prikladnoi Mekhaniki i Tekhnicheskoi Fiziki, No. 6, pp. 91-98, November-December, 1977] 806-811.," 1978.

[6] J. Ortiz-Osorio, J. M. MacInnes, P. J. Jordan, G. H. Priestman, and R. W. K. Allen, "Computation of Mass transfer in Rotating Spiral Microchannel Distillation," in The Eighth World Congress of Chemical Engineering, Montreal, July 2009.

[7] J. M. MacInnes, M. J. Pitt, G. H. Priestman, and R. W. K. Allen, "Analysis of two-phase contacting in a rotating spiral channel," Chem. Eng. Sci., vol. 69, pp. 304-315, 2012.

[8] A. A. Ayash and J. M. MacInnes, "Mass Transfer Prediction of Gas-Liquid Contacting in a Rotating Spiral Channel," in the 4th International Conference of Fluid Flow, Heat and Mass Transfer (FFHMT'17), 2017, no. 132, pp. 1-8.

[9] A. A. Ayash, "Counter-current gas-liquid contacting in a Rotating Spiral Channel: Experimental and Theoretical Study," Sheffield, 2018.

[10] J. Comyn, L. Mascia, G. Xiao, and B. M. Parker, "Corona-discharge treatment of polyetheretherketone (PEEK) for adhesive bonding," Int. J. Adhes. Adhes., vol. 16, no. 4, pp. 301-304, 1996.

[11] P. B. Kowalczuk and J. Drzymala, "Some remarks on attachment of a gas bubble to another phase both immersed in water," Physicochem. Probl. Miner. Process., vol. 52, no. 1, pp. 147-154, 2016. 